University of Nebraska - Lincoln

DigitalCommons@University of Nebraska - Lincoln

Industrial and Management Systems

Industrial and Management Systems

Engineering Faculty Publications

Engineering

2000

\title{
Quality Inspection Task in Modern Manufacturing
}

J. A. Pesante-Santana

Polytechnic University of Puerto Rico

Jeffrey C. Woldstad

University of Nebraska - Lincoln, Jeffrey.Woldstad@sdsmt.edu

Follow this and additional works at: https://digitalcommons.unl.edu/imsefacpub

Part of the Operations Research, Systems Engineering and Industrial Engineering Commons

Pesante-Santana, J. A. and Woldstad, Jeffrey C., "Quality Inspection Task in Modern Manufacturing" (2000). Industrial and Management Systems Engineering Faculty Publications. 11.

https://digitalcommons.unl.edu/imsefacpub/11

This Article is brought to you for free and open access by the Industrial and Management Systems Engineering at DigitalCommons@University of Nebraska - Lincoln. It has been accepted for inclusion in Industrial and Management Systems Engineering Faculty Publications by an authorized administrator of DigitalCommons@University of Nebraska - Lincoln. 


\title{
Quality Inspection Task in Modern Manufacturing
}

\author{
J. A. Pesante-Santana* and J. C. Woldstad ${ }^{\dagger}$ \\ * Department of Industrial Engineering, Polytechnic University of Puerto Rico, San Juan, Puerto Rico 00919-2017 \\ † Department of Industrial Engineering, Texas Tech University, Lubbock, TX 79409-3061, USA
}

\section{Introduction}

The development of numerically controlled machines, group technology, cellular manufacturing and just-intime (JIT) production systems have revolutionized the way products are designed and manufactured. These technological and strategic advances have changed the role of human operators in the manufacturing environment. The highly specialized work force of the low-tech manufacturing system has evolved into the multi-skilled work force of the high- tech manufacturing system. Among the multiple tasks that an operator is expected to perform in advance manufacturing systems (AMS) are job scheduling, inventory planning, machine set-up, problem-solving and quality inspection.

Throughout this evolution, human sensory detection capabilities have been a vital but often ignored component of the quality inspection task. Although automation is often employed to construct and assemble products within AMS, most inspections and quality checks are still done by human operators due to the inherent problems in machine vision and decision-making. While humans remain responsible for inspection, it has been widely accepted that the quality inspection task performed by humans is prone to error. Some studies indicate human inspectors typically find only $\sim 80 \%$ of the defects. Despite the contributions of human factors research to the understanding of human performance in the quality inspection task, the manufacturing trend has been to design quality schemes that compensate for poor inspector performance instead of trying to improve it (Drury 1992).

\section{A Visual Quality Inspection Taxonomy}

Quality is usually defined as fitness for use, or the extent to which a product meets the consumer requirements. Inspection is the act of measuring or examining carefully the quality of a product. Sensory inspections (performed by means of the human senses to assess a product's qualitative characteristics) and physical in- spections (performed by means of measuring devices to assess a product's quantitative characteristics) are the main types of quality inspections. The visual quality inspection task has been described as consisting of the following subtasks (Wang and Drury 1989): (1) orient the item, (2) search the item, (3) detect any defect, (4) recognize/classify the defect, (5) decide the status of the item, (6) dispatch the item, and (7) record the information about the item. These sub-tasks can be combined into two main components: search and decision-making. Thus, the simplest description of the visual quality inspection task is to search, recognize a defect and make a decision on the part's acceptability within the quality limits.

\subsection{The Visual Search Components}

Visual search is a sequential process that proceeds as a series of fixations linked, by eye movements and which terminates upon successful detection of a defect or the complete inspection of the unit. It has been shown that almost all of the information in a visual search is obtained during the fixations, which account for $>90 \%$ of the search time. In the inspector's field of view, a defect is only visible within a limited area referred to as the visual lobe. During a fixation the visual lobe is located around the central fixation point. The visual lobe size wilt be affected by the luminance of the object inspected, the contrast between the object and the defect on the object, the defect size and the distance of the defect from the inspector's eyes. Megaw and Richardson (1979) conducted eye movement studies of inspectors and concluded that inspectors do not follow a simple pattern or strategy in searching an object. They observed that while a very random appearing search pattern was used for the inspection of complex units (e.g. circuit boards), a more systematic search pattern was used for the inspection of simpler ones (e.g. knitwear).

In addition to the lobe size and the search strategy, the time available for the inspection will affect human performance in the visual search component of the in- 
spection. The more time the inspector has to search, the better the chances are of finding the defect. For the visual search subtask, the best inspectors are those who use fewer, longer fixations, as compared with those with a larger number of shorter duration fixations.

\subsection{Decision-Making Component}

Given its strict relevance to decision-making, signal detection theory (SDT) has been used to explain the decision- making component of the quality control inspection task. In a quality inspection context, SDT proposes that the human, functioning as a defect detection device, builds up in the neural system two distributions of activity: one relating to the probability of accepting a unit, the other to the probability of rejecting it. The degree of separation of these two distributions' means is a measure of the inspector's discriminability of the defects $\left(d^{\prime}\right)$. The criterion level $(\beta)$, which is the ratio of the two ordinates of the curves at a given level $X_{c^{\prime}}$ delineates the boundary between accepting and rejecting a unit, and in doing so takes in some good units to be rejected and some faulty units to be accepted. Inspectors make a correct decision either by accepting a good unit (correct rejection) or by rejecting an unacceptable unit (hit). They fail either by not detecting a rejectable defect (miss) or by falsely reporting the presence of a rejectable defect (false alarm).

Both decision-making-performance measures $\left(d^{\prime}\right.$ and $\beta$ ) are derived from the hit rate and the false alarm rate. The pure decision-making component of the quality inspection task can be measured by concentrating on tasks that require no search. The general conclusion of quality inspection studies reviewed by Drury and Fox (1975) is that "the decision-making component is among those rare tasks where a human being behaves like a rational economic decision maker, balancing the costs and payoffs involved to arrive at an optimum performance." As a normative model, SDT defines the optimal criterion $\left(\beta_{\text {opt }}\right)$ used by the ideal observer to optimize economic gains. Based on the values of a hit and a correct rejection, and the costs of a miss and a false alarm, $\beta_{\text {obs }}$ of a rational observer can be calculated and compared with that of the theoretical ideal observer $\left(\beta_{\text {opt }}\right)$. After comparing $\beta_{\text {obs }}$ with $\beta_{\text {opt }^{\prime}}$ it has been generally found that rational observers do fairly well at optimizing their winnings. However, they tend not to set extremely low or high criterion, even in situations where these strategies would lead to optimal performance.

\section{Signal Detection Theory Models of Inspection}

SDT was first used to model the decision-making performance of the quality inspection task by Wallack and Adams in 1969. After using SDT to study the performance of industrial electronics inspectors in a visual, subjectpaced task, they concluded that SDT performance mea- sures $\left(d^{\prime}, \beta\right)$ were more useful than the other available measures. Wallack and Adams concluded that SDT is useful because in addition to relating performance to payoff, it also indicates the magnitude and the direction of improvement required. Although not all of the research using SDT to study the decision-making performance has been conducted in an industrial inspection context, its findings have been beneficial in understanding human quality inspector performance.

More recently another measure of criterion level or decision-making response bias called index $c$ has been developed. The main difference between $\beta$ and index $c$ is in the way these bias indices locate the criterion $\left(X_{c}\right)$. The likelihood ratio measure, $\beta$, locates $X_{c}$ by the ratio of the heights of the SDT distributions (NSN), while index $c$ locates $X_{c}$ by its distance from the intersection of the two distributions. The range of $c$ is, therefore, the same as that of $d^{\prime}$, although zero is at the center rather than an endpoint. This parametric index is considered to be more effective than $\beta$ over a full range of sensitivity in recognition memory experiments and in vigilance. Unlike index $c, \beta$ has a tendency to produce extremely high values for conservative observers resulting in a scale that does not produce equal intervals. Index $c$ is generally more sensitive than $\beta$ to the nonperceptual manipulations of signal probability, payoff and probability shifts. Also, $\beta$ is less effective than $c$ at differentiating variations in response bias tendencies when sensitivity approached chance.

In most if not all of the research using SDT, the quality inspection task has been characterized as a vigilance situation in which the inspector's sole task is to examine a stream of products to detect and remove the defective ones. This characterization is no longer consistent with the reality of the operator's responsibilities in modern manufacturing, also known as advanced manufacturing systems (AMS). The quality inspection task in AMS is no longer a specialized task; instead, it is one of multiple dissimilar tasks conducted by a highly skilled operator. Recent research indicated that the performance of the operator in the quality inspection task while multitasking in an AMS will be determined by the interaction between the number of different types of defects that can be presented at the same time in the inspected parts and multitasking (Pesante-Santana 1997).

\subsection{Changes Over Time in the SDT Parameters}

Based on the review of 12 studies conducted between 1969 and 1975, Swets (1977) indicated that all 12 experiments showed an increasingly strict criterion $(\beta)$ over time when the signal-to-noise ratio was low; eight of the experiments showed a constant sensitivity $\left(d^{\prime}\right)$ over time. In the four studies in which $d^{\prime}$ did not remain constant, it was found to decrease by $20 \%$. An increase in $\beta$ (conservative criterion) represents a decrease in signals detected 
as well as a decrease in false alarm errors. In general, the performance changes over time have been characterized by a shift in the subject's response criterion $(\beta)$.

\subsection{Determinants of Decision-Making Performance}

It is often reported that the decision-making performance is affected by the payoff matrix, knowledge of results (KR) and signal ratios (fraction of defective). Conservative or stringent decision-making should be expected if the operator knows that the cost of an incorrect decision may be a disciplinary action, a. monetary penalty or job termination. A similar conservative decision should be expected if the process defective fraction is low (signal ratios), or if the operator receives information (knowledge of results) about specific defects or process conditions that needs to be detected for the benefit of the production area. When none of the conditions previously mentioned are not present a lax decision-making performance should be expected from the operator. However, it is important to recognize that a key element in the decision-making performance is the training. The operator's decision-making performance will not be consistent with the scenarios described in this section if $s /$ he does not have the appropriate training on quality inspection.

\section{Quality Inspection Training}

Training is essential to improving the decision-making performance of human operators. Many authors have reported that deficiency in knowledge (education or training) and or feedback (knowledge of results) is a cause of errors consistently made by inspectors. Drury (1992) listed five techniques that have proven effective in training for inspection: (1) cueing, (2) feedback, (3) active training, (4) progressive parts and (5) develop schema.

\section{Hybrid Inspection Systems}

Zero-defect products and shorter lead-time production are vital for the survival and success of modern manufacturing businesses in the current highly competitive world class manufacturing environment. According to Drury and Sinclair (1983), "this can often be achieved only by $100 \%$ inspection, which is known to be unreliable when performed by humans." This dilemma prompted an industry movement towards automated inspection systems. The advent of microprocessor-based automated inspection devices, at prices competitive with human inspection, called for a human factors reassessment of the human-machine function allocation possibilities in quality control (Drury and Sinclair 1983). Gramopadhye et al. (1992) proposed a framework for function allocation in inspection. They recommended accuracy, speed, flexibility and reliability as the perfor- mance criterion for the inspection system.

One of the first efforts to reassess the human-machine function allocation possibilities in quality control was conducted by Drury and Sinclair (1983). They compared the performance of experienced inspectors and a prototype optical/microprocessor inspection device. The main findings were: (1) neither the human nor the automated systems achieved an outstanding performance and (2) the automated system was better at locating the defects (search) but could not classify them as acceptable or rejectable (decision-making) as well as the human inspectors.

Using the accuracy and speed performance criterion measures combined with the false alarm rate and the hit rate, Hou et al. (1993) calculated a cost-based evaluation function. This cost-based evaluation function coupled with the Drury and Sinclair (1983) findings led to the conclusion that allocating the search function to machines and the decision-making function to humans results in better performance than pure human or pure machine inspection. This computer-search/human-decision-making system is known as a hybrid inspection system (HIS). The idea behind HIS is to capitalize on the machine speed and precision to scan the inspection unit, and on the decision-making ability exhibited by humans.

\section{Quality Inspection Task Load}

Quality inspection tasks that impose a sustained load on working memory (to recall what the quality acceptability criterion looks like) will demand the continuous supply of processing resources. Parasuraman (1979) conducted an experiment using a successive-discrimination task (which imposed a memory load) in which the signal was specified as the decrease in the intensity of a flashing light. The signals were presented irregularly at a mean rate of two signals $\mathrm{min}^{-1}$, and the event rate was 30 events $\mathrm{min}^{-1}$. The duration of the task was $45 \mathrm{~min}$. He concluded that the performance in such areas of vigilance application as radar monitoring and industrial quality inspection could be adversely affected when the operator has to discriminate a signal from a standard represented in memory and when the event rate is high. This performance decrement may result either from signal-data limits (weak signal in noise), or memory-data limits (quality of stored representation of the standard in delayed comparison memory tasks).

Like many other tasks, quality inspection has been identified as having an inverted-U-shaped relationship between task demand and performance level. The inverted- $U$ theory states that for a given task there is an optimal level of workload or demand that yields the highest level of performance. A departure in either direction from the optimal level of work will result in a performance decrement. While most of the results of 
vigilance research support the right-hand side of the inverted-U theory (overload) there is a lack of support for the left-hand branch (assertion that the task performance level can be improved by increasing the load). McGrath (1965) conducted one of the first experiments that supported the left-hand side in a vigilance task. After comparing easy and hard visual monitoring tasks conducted concurrently he concluded that the presence of the hard task facilitated performance on the easy one. Wiener et al. (1984) conducted an experiment in which a control group performed a vigilance task (the signal was the decrease in distance between two dots presented on a computer screen), and a second group performed a one-dimensional compensatory tracking task in addition to the vigilance task. They found that the performance of the second group (vigilance and tracking tasks) in terms of signal detection exceeded the performance of the control group (vigilance task only). They concluded that these research results provided support for the facilitating effect of increasing the task load (left side of the inverted-U).

Some researchers describe the quality inspection task as being intrinsically boring. According to them, this explains why it is often the case that mild stress will increase the performance in terms of detection and response time. However, Wickens (1992) has indicated that vigilance tasks with working memory loads are susceptible to interference from concurrent tasks. The results of a recent study on the effects of multitasking on the decision-making component of the quality inspection task were consistent with the information presented herein (Pesante-Santana 1997). The performance of the operator in the quality inspection task while multitasking in an AMS will be determined not only by the number of different types of defects that can be presented at a time in the inspected parts, but also by the mental processing resources required to meet the demand imposed by the multiple independent tasks and the memorized quality criterion. The best performance will be obtained when the additional tasks' load minimizes the monotony of the quality inspection task without interfering with the processing resources needed for the memorized quality criterion.

\section{Recommendations}

Whenever human operators perform the quality inspection a certain degree of error should be expected. However, some ideas oriented to minimize the quality inspection errors are:

- Identify the inspector with the best performance and understand his/her inspection strategy.

- Provide off-line training/practice on the actual task to be conducted using the strategy of the best inspector. o Provide continuous feedback to the operators on their performance.

- Assure an appropriate task allocation to avoid unwanted task loading.

- Use the appropriate payoff matrix to improve the operator's performance.

$\circ$ Let the proper process information (KR) flow among the operators.

\section{References}

Drury, C. G., 1992, Inspection performance. In Salvendy, G. (ed.) Handbook of Industrial Engineering (New York: Wiley), pp. 2282-314.

Drury, C. G. and Fox, J. G., 1975, Human Reliability in Quality Control (New York: Taylor \& Francis).

Drury, C. G. and Sinclair, M. A., 1983, Human and machine performance in an inspection task. Human Factors, 25, 391-9.

Gramopadhye, A. K., Drury, C. G., Sharit, J., and Sudit, M., 1992, A framework for function allocation in inspection. In Brodner, P. and Karwowski, W. (eds.) Ergonomics of Hybrid Automated Systems III (Amsterdam: Elsevier), pp. 249-57.

Hou, T., Lin, L., and Drury, C. G., 1993, An empirical study of hybrid inspection systems and allocation of inspection functions. International Journal of Human Factors in Manufacturing, 3, 351-67.

McGrath, J. J., 1965, Performance sharing in an audiovisual vigilance task. Human Factors, 7, 141-53.

Megaw, E. D. and Richardson, J., 1979, Eye movements and industrial inspectors. Applied Ergonomics, 10, 145-54.

Parasuraman, R., 1979, Memory load and event rate control sensitivity decrements in sustained attention. Science, 205, 925-7.

Pesante-Santana, J. A., 1997, The effects of multitasking on quality inspection in advanced manufacturing systems. Doctoral Dissertation (submitted and approved at the Industrial and Systems Engineering Department) (Blacksburg: Virginia Polytechnic Institute and State University).

Swets, J. A., 1977, Signal detection theory applied to vigilance. In Mackie, R.R. (ed.) Vigilance: Theory, Operational Performance and Physiological Correlates (New York: Plenum), pp. 705-18.

Wallack, P. M. and Adams, S. K., 1969, The utility of signal detection theory in the analysis of industrial inspector accuracy. AIIE Transactions, 1, 33-44.

Wang, M. J. and Drury, C. G., 1989, A method of evaluating inspectors performance differences and job requirements. Applied Ergonomics, 20, 181-90.

Wiener, E. L., Curry, R. E., and Faustina, M. L., 1984, Vigilance and task load: in search of the inverted U. Human Factors, $26,215-22$.

Wickens, C. D., 1992, Engineering Psychology and Human Performance (New York: Harper Collins). 